\title{
Synchronization in driven versus autonomous coupled chaotic maps
}

\author{
M. Pineda ${ }^{1}$ and M. G. Cosenza ${ }^{2}$ \\ ${ }^{1}$ Max-Planck-Institut für Physik Komplexer Systeme, Nöthnizer Strasse 38, 01187 Dresden, Germany \\ ${ }^{2}$ Centro de Física Fundamental, Universidad de Los Andes, Apartado Postal 26, Mérida 5251, Venezuela.
}

\begin{abstract}
The phenomenon of synchronization occurring in a locally coupled map lattice subject to an external drive is compared to the synchronization process in an autonomous coupled map system with similar local couplings plus a global interaction. It is shown that chaotic synchronized states in both systems are equivalent, but the collective states arising after the chaotic synchronized state becomes unstable can be different in these two systems. It is found that the external drive induces chaotic synchronization as well as synchronization of unstable periodic orbits of the local dynamics in the driven lattice. On the other hand, the addition of a global interaction in the autonomous system allows for chaotic synchronization that is not possible in a large coupled map system possessing only local couplings.
\end{abstract}

PACS numbers: 05.45.-a, 89.75.Kd

Coupled map lattices have provided fruitful theoretical and computationally efficient models for investigating a variety of processes in spatially distributed dynamical systems, such as synchronization, pattern formation, phase separation, turbulence, nontrivial collective behavior, etc. [1] . Recently, the phenomena of synchronization and pattern formation induced by external forcing on spatiotemporal dynamical systems, such as chemical reactions 2, 3, 4] have been the focus of much attention. There has also been interest in experimental investigations of spontaneous pattern formation and emergence of synchronization in spatially extended systems of interacting dynamical elements, such as one-dimensional arrays of electrochemical oscillators [5], chemical and hydrodynamical systems with global coupling [6, 7], and populations of chaotic electrochemical cells having both local and global interactions [8]. The relationship between forced spatiotemporal systems and autonomous dynamical systems possessing global interactions has recently been explored in the framework of coupled map lattices [9]. In this paper, we investigate the emergence of synchronization in forced spatiotemporal systems by using a model of a coupled chaotic map lattice subjected to an external drive. We show that the chaotic synchronized state in this lattice is analogous to the chaotic synchronized state emerging in an autonomous coupled map system having similar local couplings plus a global interaction, but other collective states occur differently in these two systems. We also show that the addition of a global interaction in the autonomous system allows for chaotic synchronization that is not possible in a large coupled map system possessing only local couplings.

As a model of a driven spatiotemporal system, we consider a one-dimensional coupled map lattice subjected to a uniform external drive [9],

$$
\begin{aligned}
x_{t+1}^{i} & =\left(1-\epsilon_{2}\right) f\left(x_{t}^{i}\right) \\
& +\frac{\epsilon_{1}}{2}\left[\left(f\left(x_{t}^{i+1}\right)+f\left(x_{t}^{i-1}\right)-2 f\left(x_{t}^{i}\right)\right]+\epsilon_{2} F_{t} .\right.
\end{aligned}
$$

where $x_{t}^{i}$ is the state of element $i,(i=1,2, \ldots, N)$ at discrete time $t ; N$ is the number of oscillators; $f(x)$ de- scribes the local chaotic dynamics; $\epsilon_{1}$ measures the local diffusive coupling, $\epsilon_{2}$ expresses the coupling to the external forcing, and $F_{t}$ is the uniform driving term which can be any function of time.

The dynamics of the driven lattice can be compared with that of an autonomous spatiotemporal system, described by the following coupled map system possessing both, local and global interactions,

$$
\begin{aligned}
x_{t+1}^{i} & =\left(1-\epsilon_{2}\right) f\left(x_{t}^{i}\right)+\frac{\epsilon_{1}}{2}\left[\left(f\left(x_{t}^{i+1}\right)+f\left(x_{t}^{i-1}\right)\right.\right. \\
& \left.-2 f\left(x_{t}^{i}\right)\right]+\frac{\epsilon_{2}}{N} \sum_{i=1}^{N} f\left(x_{t}^{i}\right),
\end{aligned}
$$

where $f(x)$ is the same local map as in Eq. (11); $\epsilon_{1}$ and $\epsilon_{2}$ are the local and global coupling parameters, respectively; and the global interaction is provided by the mean field of the system. Periodic boundary conditions are assumed in both systems, Eq. (11) and Eq. (2) .

When the autonomous coupled map system in Eq. (2) reaches a synchronized state at some parameter values, the evolution of its mean field is identical to the dynamics of any local map. Thus for the same set of parameters, the driven lattice, Eq. (1), subjected to a forcing $F_{t}$ equal to the local map should exhibit a synchronized state similar to that of the associated autonomous coupled map system Eq. (2) .

The driven lattice, Eq. (1), can be expressed in vector form as

$$
\mathbf{x}_{t+1}=\left[\left(1-\epsilon_{2}\right) I+\frac{\epsilon_{1}}{2} L\right] \mathbf{f}\left(\mathbf{x}_{t}\right)+\epsilon_{2} \mathbf{F}_{t},
$$

where the vector components are $\left[\mathbf{x}_{t}\right]_{i}=x_{t}^{i},\left[\mathbf{f}\left(\mathbf{x}_{t}\right)\right]_{i}=$ $f\left(x_{t}^{i}\right)$ and $\left[\mathbf{F}_{t}\right]_{i}=F_{t} ; I$ is the $N \times N$ identity matrix, and $L$ is the $N \times N$ matrix expressing the diffusive coupling among the elements, with components given by $L_{i} i \pm 1=$ $1, L_{i i}=-2$, and $L_{i j}=0$, otherwise.

The driven lattice may reach different asymptotic spatiotemporal patterns, ordered, synchronized, or turbulent, depending on the characteristics of the drive function $F_{t}$, and on the initial conditions [9]. Here we consider synchronized states of the lattice induced by a periodic or 
a chaotic drive. A synchronized state at time $t$ is defined by the condition $x_{t}^{i}=x_{t}, \forall i$. In a synchronized state, the driven system must satisfy

$$
x_{t+1}=\left(1-\epsilon_{2}\right) f\left(x_{t}\right)+\epsilon_{2} F_{t} .
$$

The linear stability analysis of the synchronized state in the driven system leads to the condition

$$
\left|\left(1-\epsilon_{2}+\frac{\epsilon_{1}}{2} \nu_{j}\right) e^{\lambda}\right|<1,
$$

where $\nu_{j}=-4 \sin ^{2}(\pi j / N), j=0,1,2, \ldots, N-1$, is the set of eigenvalues of the coupling matrix $L$, with $N / 2$ distinct eigenvalues and each being doubly degenerate 10]; and $\lambda=\lim _{T \rightarrow \infty} \frac{1}{T} \sum_{t=0}^{T-1} \log \left|f^{\prime}\left(x_{t}\right)\right|$ is the Lyapunov exponent of the local map. Thus the range of the parameter $\epsilon_{2}$ for which a synchronized state is stable corresponds to

$$
1-2 \epsilon_{1} \sin ^{2}\left(\frac{\pi j}{N}\right)-e^{-\lambda}<\epsilon_{2}<1-2 \epsilon_{1} \sin ^{2}\left(\frac{\pi j}{N}\right)+e^{-\lambda} .
$$

In particular, in a synchronized periodic state all the elements follow the same cyclic sequence of values. Consider, for example, an orbit of period $p$ of the local map, defined by $f^{(p)}\left(\bar{x}_{n}\right)=\bar{x}_{n}$, where $\left\{\bar{x}_{1}, \bar{x}_{2}, \ldots, \bar{x}_{p}\right\}$ are the set of consecutive points belonging to the orbit, satisfying $f\left(\bar{x}_{n}\right)=\bar{x}_{n+1}, f\left(\bar{x}_{p}\right)=\bar{x}_{1}$. This periodic orbit is unstable if $e^{\lambda}=\prod_{n=1}^{p}\left|f^{\prime}\left(\bar{x}_{n}\right)\right|>1$. If an unstable periodic orbit gets synchronized in the driven lattice, then $x_{t+1}=\bar{x}_{n+1}$ and $x_{t}=\bar{x}_{n}$, and Eqs. (4) yield the solution $F_{t}=\bar{x}_{n+1}$. Thus, if the external drive follows a periodic unstable orbit of the local map, i.e., $F_{t}=\left\{\bar{x}_{1}, \bar{x}_{2}, \ldots, \bar{x}_{p}\right\}$, then it is possible to synchronize the entire lattice on that orbit.

As an application, we shall consider a logarithmic map 11] $f(x)=b-\ln |x|, x \in(-\infty, \infty)$, as local chaotic dynamics in both Eq. (11) and Eq. (2). This map exhibits robust chaos, with no periodic windows and no separated chaotic bands, in the parameter interval $b \in[-1,1]$.

In order to characterize the collective states in both systems, we calculate the mean field

$$
h_{t}=\frac{1}{N} \sum_{i=1}^{N} f\left(x_{t}^{i}\right) .
$$

Figures 1(a)-1(c) show bifurcation diagrams of $h_{t}$ as a function of the coupling drive parameter $\epsilon_{2}$ for lattices driven with different forms of $F_{t}$. For each value of $\epsilon_{2}$, $h_{t}$ was calculated at each time step during a run starting from random initial conditions on the local maps, uniformly distributed on the interval $[-8,4]$, after discarding the transients. Figure 1(a) shows the bifurcation diagram of $h_{t}$ vs. $\epsilon_{2}$ for a lattice driven with a constant term $F_{t}=\bar{x}_{1}=-0.855$, where $\bar{x}_{1}=f\left(\bar{x}_{1}\right)$ is the unstable fixed point of the local logarithmic map for $b=-0.7$. In the region labeled by PS (periodic synchronization), $h_{t}$ becomes equal to $\bar{x}_{1}$, indicating that the elements in the lattice are synchronized at this stationary value. The range of $\epsilon_{2}$ for which synchronization is observed corresponds to the range predicted by the stability condition Eq. (6), for the unstable fixed point of $f$ with $p=1$. Outside the region of stationary synchronization, other types of collective behaviors can be observed in the bifurcation diagram of Fig. 1(a). In the left region labeled by CPB (collective periodic behavior), the mean field of the system driven with constant $F_{t}=\bar{x}_{1}$ experiences a collective period- 2 motion, although the local elements are chaotic and desynchronized. A collective fixed point occurs on the right CPB region. There is also a region, labeled by T (turbulence), where $h_{t}$ follows a normal statistical behavior around a mean value with fluctuations reflecting the averaging of $N$ completely desynchronized chaotic elements 12. Figure 1(b) shows $h_{t}$ vs. $\epsilon_{2}$ for the lattice driven periodically with $F_{t}=\left\{\bar{x}_{1}, \bar{x}_{2}\right\}$, where $\bar{x}_{1}=0.18, \bar{x}_{2}=-2.44$ are the points of the unstable period-2 orbit of the local logarithmic map for $b=-0.7$. In the region labeled by PS, the mean field coincides with this unstable periodic orbit, indicating that the lattice is synchronized on this orbit. This range of $\epsilon_{2}$ is predicted by condition Eq. (6) for the unstable periodic orbit of $f$ with $p=2$ for the parameter value $b=-0.7$. Periodic collective behaviors with period 2 and period 4 in $h_{t}$ arise in the regions labeled by CPB. Figure 1(c) corresponds to a chaotic driving, with $F_{t}=-0.7+\ln \left|x_{t}\right|$. Chaotic synchronization of the system occurs in the region labeled by CS and it is also predicted by Eq. (6). In this CS region, $h_{t}=F_{t}=-0.7+\ln \left|x_{t}\right|=x_{t}^{i}, \forall i$. After crossing the boundaries of the CS region, the collective states described by $h_{t}$ take the form of chaotic bands. These states are labeled CCB (collective chaotic bands) and they consist of the motion of chaotic elements that maintain some coherence.

Synchronized states, $x_{t}^{i}=x_{t}, \forall i$, can also emerge in the autonomous coupled map system Eq. (22). In order to study the stability of these states, we express Eq. (2) in vector form as

$$
\mathbf{x}_{t+1}=\left[\left(1-\epsilon_{2}\right) I+\frac{\epsilon_{1}}{2} L+\frac{\epsilon_{2}}{N} G\right] \mathbf{f}\left(\mathbf{x}_{t}\right),
$$

where the local connectivity matrix $L$ was defined in Eq. (3) and $G$ is the $N \times N$ global connectivity matrix having all its components equal to 1 .

The linear stability analysis of synchronized states in the autonomous system yields

$$
\left|\left(1-\epsilon_{2}+\frac{\epsilon_{1}}{2} \nu_{j}+\frac{\epsilon_{2}}{N} \gamma_{j}\right) e^{\lambda}\right|<1, \quad j=0,1, \ldots, N-1,
$$

where $\nu_{j}$ are the eigenvalues of the local coupling matrix $L$ defined above, and $\gamma_{j}=\delta_{o j} N$ correspond to the set of eigenvalues of $G$, with the zero eigenvalue having $(N-1)$-fold degeneracy. The range of $\epsilon_{2}$ for which synchronization takes place is

$$
1-2 \epsilon_{1} \sin ^{2}\left(\frac{\pi j}{N}\right)-e^{-\lambda}<\epsilon_{2}<1-2 \epsilon_{1} \sin ^{2}\left(\frac{\pi j}{N}\right)+e^{-\lambda},
$$



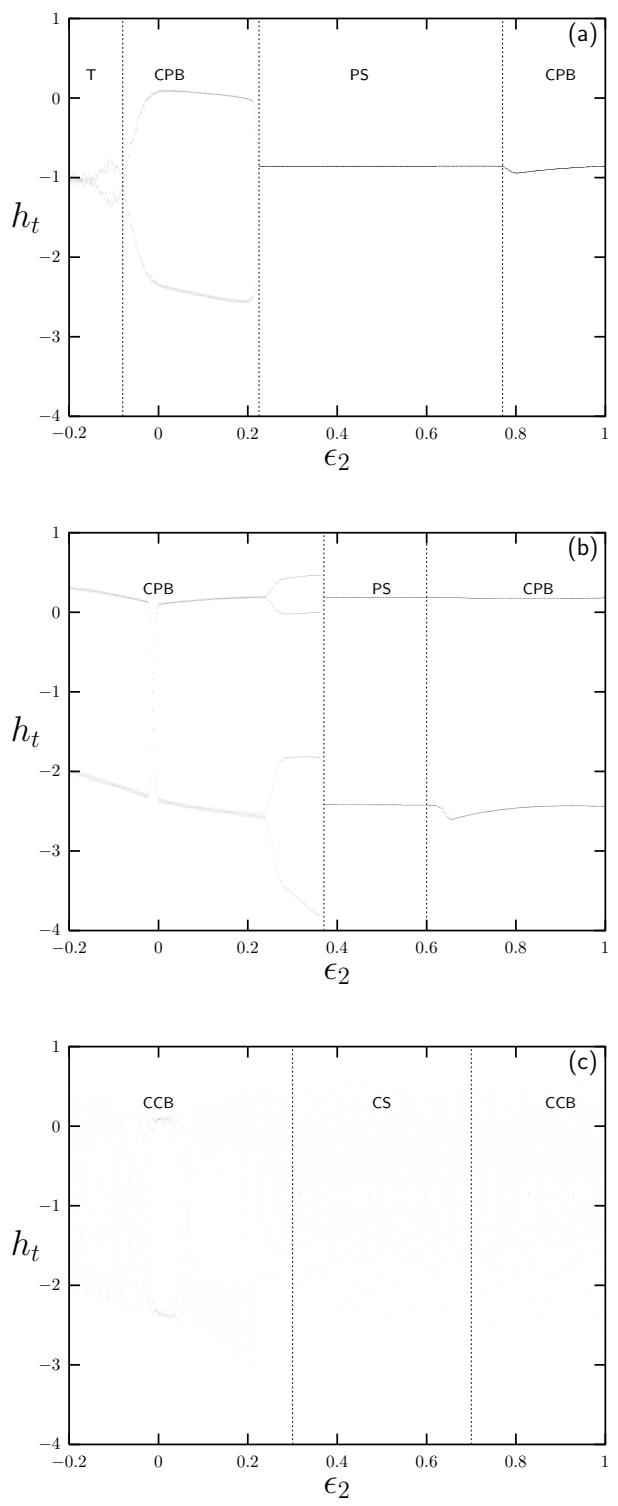

FIG. 1: Bifurcation diagrams of $h_{t}$ vs. $\epsilon_{2}$ for the driven lattice, Eq. (1), with $N=10^{4}, \epsilon_{1}=0.5, b=-0.7$. Labels indicate PS (periodic synchronization), CPB (collective periodic behavior), CS (chaotic synchronization), CCB (collective chaotic bands), and $\mathrm{T}$ (turbulence). (a) $F_{t}=\left\{\bar{x}_{1}=-0.855\right\}$. (b) $F_{t}=\left\{\bar{x}_{1}=0.18, \bar{x}_{2}=-2.44\right\}$. (c) $F_{t}=-0.7+\ln \left|x_{t}\right|$.

which is the same condition for stability of synchronized states in the driven lattice, Eq. (6). However, the unstable periodic orbits of the local map $f$ can not be synchronized in the autonomous system because they correspond to unstable synchronized states in this system.

The eigenvector corresponding to $j=0$ is homogeneous for both matrices $L$ and $G$. Thus perturbations of $\mathbf{x}_{t}$ along this eigenvector do not destroy the coherence, and the stability condition associated with $j=0$ is irrelevant for a synchronized state. The other eigenvalues corresponding to $j \neq 0$ are not homogeneous. Thus, con- dition Eq. (6] ) with $j \neq 0$ define regions in the space of parameters where all the stable synchronized states can be observed.

Figure 2 shows the boundary curves given by Eq. (9) on the parameter plane $\left(\epsilon_{2}, \epsilon_{1}\right)$, in the limit of large $N$. The label CS indicates where a chaotic synchronized state is stable in the autonomous system. After crossing the stability boundaries of the CS state, the autonomous system exhibits spatiotemporal patterns corresponding to short wave (SW) and long wave (LW) modes, as indicated in Fig. 2. Note that the stability condition Eq. (9) does not hold for $\epsilon_{2}=0$ and large $N$; i.e., chaotic synchronization for large system size $N$ can not take place in coupled map lattices possessing only local couplings, which is a well known fact [13]. Thus, the addition of a global interaction allows the emergence of chaotic synchronization in a large locally coupled system. Similarly, an external uniform driving can be used to induce chaotic synchronization in a locally coupled map network.

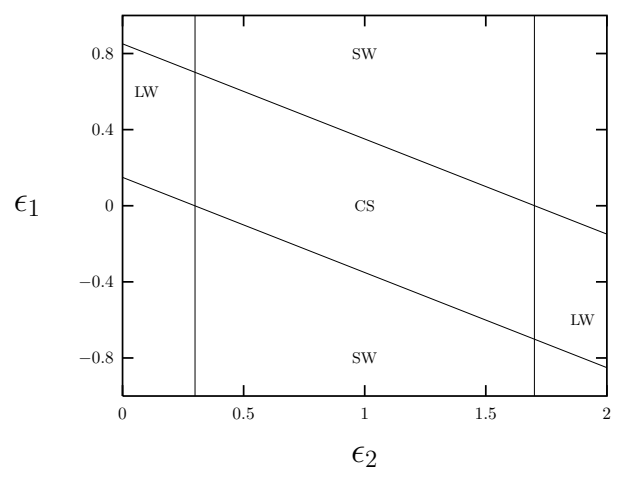

FIG. 2: Stability boundaries of the chaotic synchronized state CS of the autonomous system Eq. (2) on the parameter plane $\left(\epsilon_{2}, \epsilon_{1}\right)$, with $N=10^{4}, b=-0.7$. Regions corresponding to short wave (SW) and long wave (LW) patterns emerging from the CS state are indicated.

Figure 3(a) shows the bifurcation diagram of $h_{t}$ for the autonomous system, Eq. (21), as a function of the coupling parameter $\epsilon_{2}$. In contrast to the behavior displayed by the driven lattice, no regions of synchronization of unstable periodic orbits of the local dynamics in the space of parameters are observed in the autonomous system, as expected. The range of chaotic synchronization (CS) corresponds to the same range of $\epsilon_{2}$ for chaotic synchronization in Fig. 1(c) for the driven lattice. Note, however, that beyond the region CS in Fig. 3(a), there are collective states emerging in the autonomous system, such as CPB (collective periodic behavior), and T (turbulence), that do not appear in the corresponding diagram of the driven lattice, Fig. 1(c). By varying the coupling strengths, various spatial patterns can be realized in the autonomous system. These patterns correspond to short and to long wave modes and can be regarded as generalized Turing patterns that emerge when a global synchronized state becomes unstable [14]. Figure 3(b) shows the 

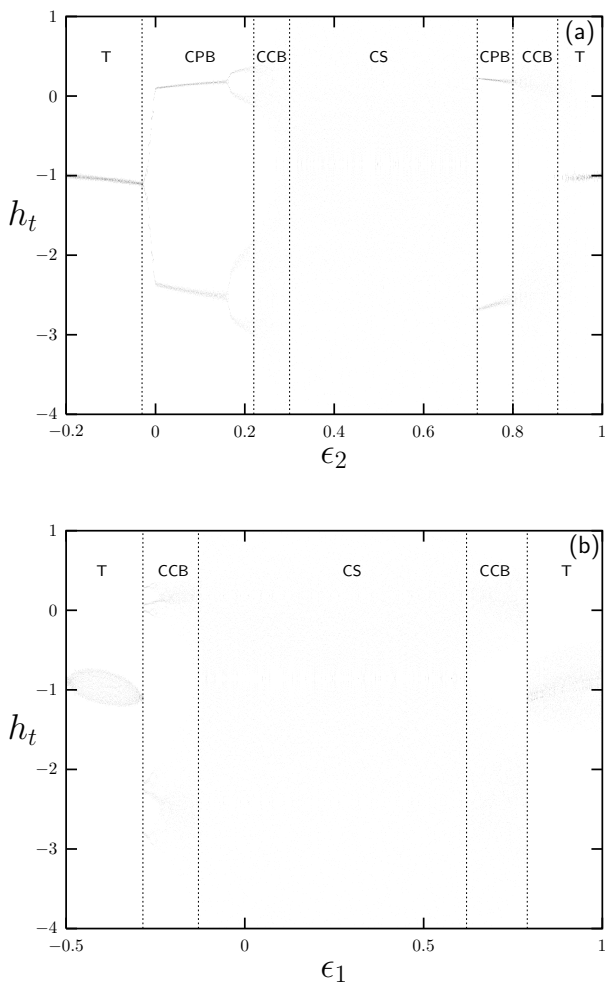

FIG. 3: Bifurcation diagrams of $h_{t}$ for the autonomous system, for $b=-0.7, N=10^{4}$. (a) $h_{t}$ vs. $\epsilon_{2}$, with fixed $\epsilon_{1}=0.5$. (b) $h_{t}$ vs. $\epsilon_{1}$, with fixed $\epsilon_{2}=0.5$. Labels are defined in Fig. 1

bifurcation diagram of $h_{t}$ vs. $\epsilon_{1}$, with $\epsilon_{2}$ fixed.

The collective states observed in the bifurcation diagrams of Fig. 3, as well as those in Fig. 1, have been checked for system-size effects. When the lattice size $N$ is increased, the segments in the CPB regions shrink, but the amplitudes of the collective periodic motions do not decrease, indicating that the global periodic attractors become better defined in the large system limit. As a consequence, the variance of the fluctuations of $h_{t}$ itself do not decrease as $N^{-1}$ with increasing $N$, but rather it saturates at some constant value related to the amplitude of the collective periodic motion. These states of ordered evolution in macroscopic quantities such as $h_{t}$, arising from local chaos in spatiotemporal systems, have been called nontrivial collective behavior [15]. The states of collective chaotic bands (CCB) are also manifestations of nontrivial collective behavior, since the variance of $h_{t}$ for those states neither follows a regular statistical behavior. In contrast, the variance of the mean field in the turbulent states (T) appears to decrease as $N^{-1}$ with increasing $N$, obeying normal statistical behavior.

In summary, we have studied the analogy between the chaotic synchronized states emerging in forced spatiotemporal systems and in autonomous dynamical systems possessing global interactions in the context of coupled map lattices. By using a model of a one-dimensional coupled map lattice subjected to a uniform external drive, we have shown that both, synchronization of unstable periodic orbits of the local maps and chaotic synchronized states, can be induced in the driven lattice. The external drive acts as a control mechanism for stabilizing unstable periodic orbits of the local maps. We showed that the synchronization behavior of the driven lattice can be compared, under some circumstances, with that of an autonomous coupled map system possessing a similar local coupling plus an additional global interaction that acts as a global feedback. It was found that the chaotic synchronized states in both systems are analogous; however, the autonomous system does not exhibit synchronization of unstable local periodic orbits. The collective states arising when the chaotic synchronized state becomes unstable are, in general, different in these systems. Although we have considered one-dimensional diffusive couplings, expressed by the matrix $L$, the analogy between a uniform external drive and a global interaction can also be applied to other networks of coupled maps whose connectivity may be represented in matrix form.

M.P. is supported by a DAAD scholarship, Germany. We thank H. Kantz and A.E. Motter for useful comments.
[1] Chaos 2, No. 3 (1992), focus issue on Coupled Map Lattices; edited by K.Kaneko.

[2] A. L. Lin, A. Hagberg, A. Ardelea, M. Bertram, H. L. Swinney, and E. Meron, Phys. Rev. E 62, 3790 (2000).

[3] W. Wang, I. Z. Kiss, and J. L. Hudson, Phys. Rev. Lett. 86, 4954 (2001).

[4] C. Hemming and R. Kapral, Physica A 306, 199 (2002).

[5] W. Wang, I. Z. Kiss, and J. L. Hudson, J. Phys. Chem. B 1032178 (1999).

[6] W. Wang, I. Z. Kiss, and J. L. Hudson, Chaos 10248 (2000).

[7] K. Miyakawa and K. Yamada, Physica D, 151, 217 (2001).

[8] I. Z. Kiss, Y. Zhai, and J. L. Hudson, Phys. Rev. Lett. 88, 238301 (2002).
[9] M. G. Cosenza, M. Pineda, and A. Parravano, Phys. Rev. E 67, 066217 (2003).

[10] I. Waller and R. Kapral, Phys. Rev. A 30, 2047 (1984).

[11] T. Kawabe and K. Kondo, Prog. Theor. Phys. 85, 759 (1991).

[12] M. G. Cosenza and J. González, Prog. Theor. Phys. 100, 21 (1998).

[13] A. Pikovsky, M. Rosenblum, and J. Kurths, Synchronization, a universal concept in nonlinear sciences, Cambrigde University Press, Cambridge, UK (2001).

[14] G. Rangarajan, Y. Chen, and M. Ding, Phys. Lett. A 310, 415 (2003).

[15] H. Chaté and P. Manneville, Prog. Theor. Phys. 87, 1 (1992). 